\title{
Modelo de competencias del profesor de medicina
}

\author{
A. Martínez-Gonzáleza , J. López-Bárcena b, P. Herrera Saint-Leuc, J. Ocampo-Martínez ', \\ I. Petra ${ }^{e}$, G. Uribe-Martínez ${ }^{\dagger}$ M.C. García-Sahagún ${ }^{\text {}}$, S. Morales-López ${ }^{9}$
}

Objetivo. Generar el modelo de competencias del profesor para ejercer la docencia de alto nivel en Medicina. Materiales y métodos. Para generar el modelo se analizó la bibliografía, el propósito del plan de estudio por competencias y se utilizó el método Developing a Curriculum (DACUM), con la participación de 35 expertos que integraron mediante consenso el trabajo que debe realizar un docente de medicina para cumplir con éxito el propósito del plan de estudio. Resultados. Se identificaron las funciones básicas de los profesores de medicina, así como las actividades para su cumplimiento. Se conformó el modelo con seis competencias: disciplinaria, de investigación, psicopedagógica, de comunicación, académicoadministrativa y humanística. Conclusiones. El modelo pretende guiar las acciones de los profesores que actualmente participan en el programa y aquellos que aspiran a formar parte del proceso educativo; elaborar programas para la formación de profesores que favorezcan el desarrollo de competencias académicas; identificar elementos que se deben considerar para la evaluación del desempeño docente; orientar el diseño de indicadores útiles para otorgar reconocimientos y estímulos a los profesores cuyo desempeño académico sea excepcional; orientar al estudiante, en la relación profesor-alumno, sobre las funciones y actividades que puede esperar del profesor.

Palabras clave. Competencias. Educación médica. Funciones. Modelo. Profesor.

\section{Competencies model of medical teacher}

Aim. To create a competency model for the medical professor in order to exert teaching at its highest level. Materials and methods. To create such model, we analyzed current literature on teaching, the aim of the curricula for competen- cies and we used the DACUM method whose letters stand for Developing a Curriculum. 35 experts who participated in this project reached a consensus regarding the duties that have to be carried out by a medical professor in order to successfully fulfill the aim of the curricula. Results. We identified the basic functions of medical professors as well as the related duties for its fulfillment. The model comprises six competencies: disciplinary, research, psico-pedagogy, communicative, academic-administrative and humanistic competencies. Conclusion. This model pretends to guide the actions of medical professors who are currently participating in this program and those who want to become part of the education process. It also pretends to create programs favoring the professors 'training benefiting the development of academic competencies; to identify the elements to be considered for the assessment of the professors ' development; to guide the design of useful indicators to grant awards and incentives to those professors whose academic development be exceptional; to guide the student within the professor-student relationship, on the duties and activities that they can expect from the professor.

Key words. Competencies. Functions. Medical education. Model. Profesor.

\section{Introducción}

La enseñanza y el aprendizaje son dos elementos inseparables e integrantes de un proceso único y complejo en el que intervienen múltiples factores. En este proceso los profesores juegan un papel fundamental e insustituible y asumen funciones y actividades diferentes que hacen posible la misión de la institución educativa. Se conside- a Departamento de Salud

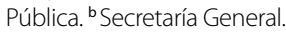
cDepartamento de Anatomía. ¿Departamento de Historia y Filosofía de la Medicina. e Departamento de Psicología Médica, Psiquiatría y Salud Mental. 'Secretaría de Enseñanza Clínica. ${ }^{9}$ Coordinación de Ciencias Básicas. Facultad de Medicina. Universidad Nacional Autónoma de

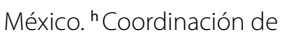
investigación y desarrollo en calidad. IMSS. México DF, México.

\section{Correspondencia Dr. Adrián Martínez González. Facultad de Medicina. UNAM. Cañaverales, 60. Casa 18. Col. Rinconada Coapa del Tlalpan. CP 14330 México DF, México. \\ E-mail aamg@servidor.unam.mx}

\section{Agradecimientos A todos los profesores que participaron en el taller PAPIME (clave: PE200106) por su apoyo financiero parcial para la realización de este trabajo.}

Aceptación definitiva 08.05.2008. 
ra profesor a un individuo que utiliza distintos métodos y técnicas en presencia de un inexperto (alumno) con el fin de facilitar el aprendizaje y la elaboración de su propio conocimiento. El elemento fundamental de este proceso es la realimentación bidireccional entre el profesor y el alumno, a diferencia de una simple transmisión de información [1].

En este proceso interactúan: el estudiante, el profesor y la institución [2]. Actualmente, esta interacción se basa en un esfuerzo cooperativo, donde el profesor establece las metas de desempeño y favorece un ambiente adecuado para el aprendizaje dentro del marco que la institución provee. Por su parte, el estudiante asume la responsabilidad de su propio conocimiento y de un ejercicio pleno de su autonomía para llevar a cabo un aprendizaje más profundo y significativo [2-4].

Son escasos los autores que hacen referencia a las competencias académicas que debe poseer un buen profesor en el área médica. Entre ellos, Nogueira et al [5] establecen las competencias docentes básicas, las cuales son: preparación docente sistemática; dominio de los contenidos que se deben impartir; selección de los métodos de enseñanza según el contenido y el tipo de clase; comprensión de los objetivos que deben lograr los estudiantes; tratamiento ético en las situaciones de enseñanza-aprendizaje; utilización de una comunicación que permita el aprendizaje; relación de los objetivos con la evaluación; organización del grupo de estudiantes, y participación del estudiante en su propio aprendizaje.

Harden y Crosby [6] identifican los roles principales que debe desempeñar el 'buen profesor'. Se presume que para hacerlo deberá tener ciertas competencias sin enunciarlas. Estos autores describen seis roles o funciones del maestro que resumen de esta manera: el maestro como proveedor de información, como modelo que se debe seguir, como administrador, como asesor, como facilitador y como generador de recursos.

Zabar et al [7] se inclinan por la evaluación de las competencias de los residentes como educadores médicos, aunque no especifican la fuente desde la cual identifican las competencias académicas de los profesores.

En Estados Unidos, algunos autores [8] analizaron nueve de los cursos para la formación de profesores que existen, y encontraron las siguientes características comunes: preparar al profesor para dirigir, enseñar y evaluar las iniciativas educativas; motivación; prácticas de aprendizaje activo; recursos electrónicos, y comunicación.

Otros autores agregan a lo anterior temas como: aplicación de las teorías del aprendizaje en el adulto en un ambiente clínico, la relevancia de las humanidades en la educación médica, habilidades de la autorreflexión y autorregulación, métodos de enseñanza, desarrollo curricular, evaluación del aprendizaje, liderazgo y cambio organizacional e investigación educativa $[9,10]$.

Searle [11] incluye además al profesor como modelo, que debe considerar las necesidades del aprendiz, como son la buena retroalimentación/comunicación, especialmente en el área de la clínica.

Las competencias profesionales de los médicos generales son un tema central dentro de la educación médica debido a que son la base para el desarrollo de planes de estudio vigentes en varias universidades y que contribuyen a la inminente globalización de esta profesión, lo que obliga a que los estándares y los requerimientos de los médicos deban ser similares en todo el mundo. La Facultad de Medicina de la Universidad Nacional Autónoma de México (UNAM) ha definido como un elemento central en su futuro plan de estudios las competencias que debe poseer un licenciado de la carrera de Médico Cirujano para que éste sea capaz de cumplir con los más altos estándares mundial y regionalmente. El proceso de formación de los médicos es largo y complejo. En su parte medular, implica la asimilación por parte del estudiante de conocimientos científicos, habilidades, actitudes y valores. Además del adiestramiento multidisciplinario requerido y de las competencias clínicas, la formación debe contemplar ciertas estrategias, bajo la guía y supervisión de un conjunto de profesores experimentados para que el estudiante adquiera, de manera progresiva, la capacidad de solucionar problemas de forma independiente de acuerdo con los perfiles de licenciado y profesional para un desempeño exitoso en la sociedad contemporánea.

De ahí se desprende que, para lograr este propósito, el profesor tiene un papel fundamental debido a que sus funciones incluyen no sólo la provisión de información sino que participa como asesor, facilitador, creador de recursos y modelo de los futuros médicos. De acuerdo a cómo lo establecen algunos autores [3,6,12-14], el buen 
'maestro' no nace, se forma; como consecuencia, la identificación del perfil de competencias necesarias en el profesor de educación médica parte de las evaluaciones de la carrera de médico cirujano, como es el caso del informe presentado por la comisión revisora del plan de estudio en la Facultad de Medicina de la UNAM, que establece que la falta de homogeneidad en la calidad del proceso educativo da como resultado un perfil heterogéneo entre los licenciados [15]. En general, los profesores de medicina carecen de una verdadera formación docente [16]. El desconocimiento de las competencias y la poca formación pedagógica reduce la posibilidad de que los profesores de medicina cumplan cabalmente las funciones propias de un profesor. Impide la selección razonada de los aspirantes a formar parte del personal docente, dificulta la identificación de criterios para fundamentar las actividades de formación de profesores, evita la detección de adecuadas prácticas docentes y reduce el rigor de la evaluación del desempeño del profesor.

El propósito del presente estudio es generar el modelo de competencias de los profesores de medicina, así como la identificación de sus funciones, actividades y el perfil de competencias necesarias para ejercer una docencia de alto nivel.

Se suele utilizar el término modelo para hacer referencia a la representación física de un objeto, o bien como un símil material o abstracto de una situación o fenómeno. En el proceso de investigación científica se emplea como marco referencial el 'modelo conceptual'. En este sentido, se define éste como la conceptuación de un sistema [17]. Pero también se concibe en términos analógicos, es decir, como objeto destinado a reproducir fielmente la configuración y la red de relaciones entre diferentes elementos de una estructura, o bien en términos teoréticos, como una reconceptuación de un sistema específico, parcialmente conocido, sobre la base de tramas de propiedades o relaciones de otro sistema mejor conocido [19]. Si consideramos las acepciones anteriores, en este trabajo se utiliza el término modelo como la representación de una estructura abierta, dinámica y flexible de un sistema docente.

Para los fines de este trabajo se acepta que las competencias se refieren a un conjunto de conocimientos, habilidades, actitudes y valores que posee el profesor y que relacionados entre sí permiten el desempeño exitoso de las activi- dades y funciones del proceso educativo, según los indicadores y estándares establecidos, con la finalidad de conducir al estudiante a la toma de decisiones correcta y a la resolución de problemas de salud individual y colectiva basados en la evidencia.

\section{Materiales y métodos}

En el presente estudio participaron 29 profesores que conformaron el grupo interno y otros 6 profesores que integraron el grupo externo. Para definir las funciones, actividades y competencias académicas de éstos, se utilizó el método DACUM [19-23], que aprovecha la experiencia de expertos en un tema en cuestión para describir las funciones que debe realizar una persona en un trabajo o en un área ocupacional determinada, definir las competencias requeridas, determinar las necesidades de formación de los recursos humanos, establecer formas de evaluación del desempeño profesional, proponer un programa de desarrollo basado en competencias y proponer el desarrollo curricular. El producto del proceso DACUM es un perfil de competencias, funciones y actividades de una profesión específica.

Las premisas básicas del método DACUM aplicadas al campo educativo se pueden resumir de la siguiente manera: a) los profesores conocen muy bien su trabajo, por tanto, lo pueden describir mejor que nadie; b) un modo efectivo de precisar el trabajo que llevan a cabo los profesores es describir con detalle las actividades que realizan, y c) las funciones básicas de los profesores demandan ciertos conocimientos, habilidades, actitudes y valores.

Esta investigación fue de carácter cualitativo con un análisis detallado de los elementos integradores de la función docente.

Se creó un grupo de expertos con las siguientes características: profesor de tiempo completo de la Facultad de Medicina de la UNAM de las áreas: biológica, sociomédica y clínica; con experiencia docente de más de 20 años; que hubieran participado en el diseño del plan de estudios por competencias y que aceptaron integrarse voluntariamente al estudio.

Una vez creado este grupo (grupo interno), se aplicó el método DACUM en cuatro etapas: 
- Planeación. Se diseñaron los instrumentos de recopilación de información para la descripción de funciones y actividades, la definición de competencias académicas y para la validez de contenido.

- Desarrollo. Esta etapa se llevó a cabo en varias fases. La primera de ellas se desarrolló en dos sesiones de trabajo, donde, después de una breve introducción sobre los aspectos básicos del procedimiento, los coordinadores del grupo pidieron a los participantes de manera independiente: a) hacer una lista de las funciones que, a su juicio, deben desempeñar los profesores de medicina, teniendo en cuenta los objetivos primarios del plan de estudios por competencias profesionales y el perfil del licenciado; b) presentar sus propuestas al grupo de trabajo, y c) opinar sobre el trabajo realizado por los otros miembros del grupo. En la segunda fase se ordenaron las funciones descritas, y se eliminaron las repetidas. Sólo las funciones que alcanzaron un consenso por el grupo como esenciales para lograr los propósitos del plan de estudios por competencias fueron redactadas en su forma final. En la tercera fase se pidió a cada participante de manera independiente: a) describir las actividades necesarias para el cumplimiento de cada función aceptada en la fase anterior; b) presentar sus propuestas al grupo de trabajo, y c) opinar sobre el trabajo realizado por los otros miembros del grupo. En consenso, el grupo de expertos decidió cuáles son las actividades necesarias para el logro de cada una de las funciones. En la última fase, con la misma estrategia metodológica, los miembros del grupo identificaron y listaron los conocimientos, habilidades, actitudes y valores necesarios para desempeñar satisfactoriamente cada una de las funciones seleccionadas.

- Integración. Se dividió en dos fases. En la primera, el grupo externo sugirió modificar, eliminar o agregar, funciones, actividades, conocimientos, habilidades, actitudes y valores. En la segunda fase, los autores se dieron a la tarea de integrar, clasificar, redactar y conceptualizar en su caso las funciones, actividades, conocimientos, habilidades, actitudes y valores generados por los grupos interno y externo.

- Validación. El producto obtuvo el consenso de todos los participantes. Las opiniones y observaciones de ambos grupos y coordinadores fueron incluidas en el documento final.

- Definición del perfil de competencias. Se procedió al análisis y clasificación de todos los conocimientos, habilidades, actitudes y valores y a la determinación de las competencias, lo que permitió establecer el perfil de los profesores.

- Generación del modelo. Finalmente se generó el modelo de competencias de los profesores de la Facultad de Medicina de la UNAM a partir del propósito del plan de estudios (perfil de competencias del licenciado), las funciones y las actividades del perfil de competencias del profesor y del análisis y revisión de la literatura ad hoc.

\section{Resultados}

Los grupos de expertos identificaron ocho funciones básicas de los profesores de medicina, cuyo cumplimiento requiere de 49 actividades específicas. Estas funciones buscan guiar, evaluar, motivar, propiciar, estimular, facilitar, propiciar y desarrollar en el alumno el logro del perfil profesional del licenciado, asociadas al desempeño docente. Las actividades, por su parte, se dirigen a explicar cómo llevar a cabo cada una de las funciones señaladas (Tabla I).

Las seis competencias identificadas por el grupo de expertos derivadas de las funciones $y$ actividades fueron conceptualizadas de la siguiente manera:

- Disciplinarias. Incluye el dominio actualizado de su campo de conocimiento y saberes fundamentales relacionados con otras disciplinas, aplicadas a la solución de problemas de salud individuales y colectivos, que permiten la formación del estudiante para una práctica profesional autónoma, acorde con el perfil de licenciado y el perfil profesional del médico.

- Investigación. El docente utiliza la metodología científica y sustenta la práctica docente y profesional en la mejor evidencia disponible, para promover el pensamiento lógico, el desarrollo del juicio crítico del estudiante y su aplicación en la toma de decisiones ante los problemas de salud. 
Tabla I. Funciones y actividades del profesor de medicina.

\begin{tabular}{|c|c|}
\hline Funciones & Actividades \\
\hline $\begin{array}{l}\text { 1. Guiar al estudiante } \\
\text { en la identificación } \\
\text { y solución de los } \\
\text { problemas de salud } \\
\text { individuales, familiares } \\
\text { y colectivos de } \\
\text { mayor prevalencia y } \\
\text { trascendencia en el } \\
\text { país }\end{array}$ & $\begin{array}{l}\text { 1.1. Discutir los criterios para reconocer y determinar cuáles son los problemas } \\
\text { de salud individuales y colectivos prioritarios en el país y en el mundo } \\
\text { (prevalencia y trascendencia). }\end{array}$ \\
\hline
\end{tabular}

2. Evaluar en el alumno, de forma continua y objetiva, el avance y el logro de las competencias del Plan de Estudios de la carrera de Médico Cirujano

2.1. Discutir con el alumno los criterios con los cuales será evaluado.

2.2. Elaborar los instrumentos, procedimientos y técnicas necesarios que midan el grado de conocimientos, habilidades y actitudes acordes con el plan de estudios y el nivel definido.

2.3. Participar en la evaluación de la validez y confiabilidad de los instrumentos, procedimientos y técnicas aplicadas.

2.4. Analizar con el alumno los avances y las deficiencias en el proceso del aprendizaje y establecer las medidas correctivas.

2.5. Plantear diferentes procedimientos objetivos para que el alumno autoevalúe su participación en el proceso de aprendizaje y plantee estrategias y medidas de cambio.

2.6. Evaluar el desempeño de las competencias.

3. Motivar al alumno para el logro de las competencias profesionales
3.1. Desarrollar una comunicación efectiva con el alumno basada en la comprensión, el apoyo y la flexibilidad.

3.2. Fomentar la autoestima del alumno al convertir los problemas en oportunidades.

3.3. Promover en el alumno el autoconocimiento.

3.4. Detectar los aspectos emocionales y sociales que influyen sobre su rendimiento y comportamiento en grupo.

3.5. Referir a los alumnos en los que se detecten problemas académicos y psicosociales.

3.6. Formar grupos para resolver los problemas interpersonales (profesor-alumno), y aquellos relacionados con los contenidos temáticos del plan de estudios y su implementación.
4. Propiciar que el alumno desarrolle el juicio crítico con respecto a conocimientos, habilidades y actitudes, propios y ajenos, ante los problemas de salud

5. Estimular en el alumno el interés por el conocimiento biomédico, clínico, sociomédico, la investigación y la docencia
4.1. Utilizar estrategias educativas que permitan que el alumno realice autocrítica y crítica constructiva hacia los demás de forma permanente.

4.2. Cuestionar al alumno sobre el fundamento de sus aseveraciones.

4.3. Discutir criterios para discriminar la información fidedigna y confiable sobre la salud.

4.4. Plantear problemas para que el alumno discrimine los aspectos biomédicos, sociomédicos y clínicos en un problema de salud.

4.5. Elaborar y proponer modelos o ejercicios que permitan que el estudiante identifique los diferentes componentes del método científico, clínico y epidemiológico.

\author{
5.1. Relacionar el conocimiento con el proceso salud-enfermedad. \\ 5.2. Analizar las cualidades de la profesión del médico. \\ 5.3. Reflexionar sobre la función de servicio hacia la sociedad. \\ 5.4. Destacar los logros de la medicina. \\ 5.5. Organizar visitas a comunidades, centros de salud y hospitales. \\ 5.6. Involucrar a los alumnos en la discusión de problemas médicos de acuerdo \\ a su nivel académico. \\ 5.7. Facilitar el trabajo de grupo para propiciar el interés en el conocimiento médico \\ nacional e internacionalmente. \\ 5.8. Fomentar la participación en eventos académicos médicos. \\ 5.9. Promover la participación del alumno en la enseñanza y en la difusión \\ del conocimiento. \\ 5.10. Aprovechar cada problema para relacionarlo con el ámbito médico.
}




\begin{tabular}{|c|c|}
\hline Funciones & Actividades \\
\hline $\begin{array}{l}\text { 6. Facilitar que el alumno } \\
\text { logre el aprendizaje } \\
\text { autorregulado y } \\
\text { permanente de forma } \\
\text { individual y en grupo }\end{array}$ & $\begin{array}{l}\text { 6.1. Establecer estrategias que favorezcan la comunicación en el trabajo de grupos } \\
\text { colaborativos. } \\
\text { 6.2. Promover, mediante preguntas, que el alumno identifique sus necesidades } \\
\text { y problemas de aprendizaje. } \\
\text { 6.3. Analizar con los alumnos los programas de estudio para que diseñen } \\
\text { sus propias actividades. } \\
\text { 6.4 Estimular el trabajo activo del alumno para comprometerlo con sus estudios. } \\
\text { 6.5. Proporcionar al alumno estrategias de enseñanza-aprendizaje que favorezcan } \\
\text { la autorregulación. }\end{array}$ \\
\hline $\begin{array}{l}\text { 7. Propiciar que el } \\
\text { alumno adquiera y } \\
\text { mantenga una actitud } \\
\text { ética, reflexiva y } \\
\text { humanística durante } \\
\text { su formación y su } \\
\text { práctica profesional }\end{array}$ & $\begin{array}{l}\text { 7.1. Proceder de manera ética, reflexiva y humanística durante su relación } \\
\text { con los estudiantes. } \\
\text { 7.2. Reflexionar con los alumnos los problemas, situaciones y ejemplos que permitan } \\
\text { llegar a propuestas éticas y humanísticas en la disciplina correspondiente. } \\
\text { 7.3. Analizar diferentes situaciones de relación médico-paciente. } \\
\text { 7.4. Actuar frente a sus alumnos y reflexionar con ellos sobre la importancia de } \\
\text { mantener la imparcialidad ante ideologías, creencias y preferencias de etnia, } \\
\text { género y edad. }\end{array}$ \\
\hline $\begin{array}{l}\text { 8. Desarrollar de manera } \\
\text { permanente su } \\
\text { actividad docente, de } \\
\text { acuerdo a estándares } \\
\text { establecidos y } \\
\text { en función de } \\
\text { las tendencias } \\
\text { pedagógicas actuales }\end{array}$ & $\begin{array}{l}\text { 8.1. Participar en los procesos asignados para su evaluación docente } \\
\text { y realizar las acciones correctivas pertinentes. } \\
\text { 8.2. Actualizarse en su quehacer docente y en su disciplina. } \\
\text { 8.3. Integrar las nuevas tendencias pedagógicas a su acto docente. } \\
\text { 8.4. Analizar su práctica docente y reconocer fortalezas y debilidades. } \\
\text { 8.5. Planear, desarrollar y evaluar su acto docente. } \\
\text { 8.6. Llevar a cabo trabajo en equipos de pares y multidisciplinarios. } \\
\text { 8.7. Desarrollar habilidades en las relaciones interpersonales. } \\
\text { 8.8. Retroalimentar a la institución a partir de los resultados de su investigación } \\
\text { docente y del proceso de aprendizaje. }\end{array}$ \\
\hline
\end{tabular}

- Psicopedagógicas. Incluye el conocimieto suficiente de la psicología individual y de grupo, asimismo de la pedagogía y la didáctica, para desempeñarse de una manera eficiente en el ámbito académico de la medicina, lo que facilita el aprendizaje significativo del estudiante.

- Comunicación. El docente establece una comunicación interpersonal efectiva en el contexto de la práctica de la medicina general, que le permite propiciar el desarrollo y fortalecimiento de las habilidades de comunicación verbal y no verbal de los estudiantes.

- Académico-administrativas. Realiza un ejercicio docente basado en el conocimiento de las necesidades institucionales y en el cumplimiento de la misión, las normas y los programas académicos de la Facultad de Medicina.

- Humanística. Incluye el conocimiento de las humanidades médicas y la observancia de actitudes y valores éticos, que en su conjunto proporcionan una formación humanística integral y un modelo para el alumno.
La tabla II describe las competencias necesarias para el desempeño docente eficiente en medicina.

Para el logro de las competencias que corresponden a su perfil y para el desempeño de sus funciones y actividades, el profesor deberá tener como cualidades esenciales autoridad moral y vocación docente, $\mathrm{y}$ asumir actitudes sustentadas en valores epistémicos como objetividad, verdad, racionalidad y en valores morales como altruismo, honorabilidad, confianza, justicia y probidad en un contexto determinado (Tabla III).

En la figura se observa el modelo de competencias de los profesores de medicina en interacción con el contexto de la práctica docente.

\section{Discusión}

Entre los desafíos que hoy enfrentan las Escuelas y Facultades de Medicina con respecto a la formación y capacitación de profesores, se encuen- 
Tabla II. Perfil de competencias del profesor de medicina (conocimientos y habilidades).

\section{Disciplinarias}

a) Conocimientos básicos sobre:

- Las características generales de la estructura y función del ser humano.

- Las enfermedades de mayor prevalencia y trascendencia.

- Principios de la relación médico-paciente.

- Cultura general.

b) Conocimientos en:

- Los avances de su disciplina y área de conocimiento.

c) Habilidades:

- Recopilar, ordenar e interpretar la información de la historia clínica, de los estudios auxiliares de diagnóstico y epidemiológica.

- Relacionar los hechos o condiciones asociados con la salud y la enfermedad.

- Afrontar los problemas de salud mediante el análisis, síntesis y evaluación.

- Capacidad para percibir la medicina desde una visión holística.

\section{Investigación}

a) Conocimientos:

- Manejo de las fuentes de información científica.

- Método científico, clínico y epidemiológico..

b) Habilidades:

- Buscar e interpretar información científica a través del manejo adecuado del método científico.

- Discriminar la validez y confiabilidad de la información obtenida.

- Orientar al alumno en la toma de decisiones con base al juicio crítico.

- Estimular la curiosidad científica poniendo énfasis en las ventajas del conocimiento interdisciplinario.

\section{Psicopedagógicas}

a) Conocimientos:

- Las orientaciones pedagógicas y psicológicas fundamentales.

- Los avances en la educación médica.

- Los avances de los aspectos psicológicos y pedagógicos aplicables a la educación médica.

- Las diferencias psicosociales de género.

- Las estrategias educativas.

- Los recursos didácticos.

- La evaluación educativa.

- Las técnicas para el manejo de grupos.

- El proceso psicológico del adolescente y del adulto para la detección de actitudes normales y anormales del alumno.

- La asertividad.

- Las técnicas para propiciar la resiliencia.

- Las técnicas de confrontación.

b) Habilidades:

- Diseñar instrumentos de evaluación.

- Interpretar informes de evaluación.

- Retroalimentar el aprendizaje de los alumnos.

- Promover en los alumnos la autoevaluación.

- Ejercitar la crítica constructiva con los alumnos.

- Propiciar la toma de decisiones.

- Promover el desarrollo de habilidades psicosociales.

- Inducir al cuestionamiento.

- Fomentar el aprendizaje significativo con relación a la medicina.

- Promover el aprendizaje autodirigido.

- Aplicar eficientemente los recursos psicopedagógicos.

- Proporcionar a los alumnos opciones para la autorregulación de su aprendizaje. 
- Reconocer los problemas de aprendizaje en el individuo y en el grupo.

- Ser flexible en el proceso educativo.

- Adaptarse a los estilos de aprendizaje de los alumnos.

- Instrumentar las estrategias de aprendizaje adecuadas a la situación educativa.

- Inducir el intercambio de experiencias entre pares.

- Observar y analizar el proceso educativo.

- Crear condiciones para el logro del aprendizaje individual y de grupo.

- Conducir la dinámica del grupo.

- Desarrollar la introspección propia y del alumno.

- Guiar eficientemente las emociones propias y de los alumnos.

- Capacidad para motivar a los alumnos.

\section{Comunicación}

a) Conocimientos:

- La teoría de la comunicación humana.

- Las técnicas de comunicación individual y de grupo.

- Idioma español e inglés.

b) Habilidades:

- Orientar la discusión con los alumnos en un ambiente de tolerancia, respeto y ecuanimidad.

- Escuchar de forma activa.

- Manejar eficientemente el lenguaje verbal y no verbal.

- Utilizar los ¿lineamientos?? de la comunicación del conocimiento de la ciencia.

- Establecer comunicación efectiva con el estudiante, el paciente, la familia, la comunidad y los pares.

- Estimular la comprensión y la redacción del español e inglés.

\section{Académico-administrativas}

a) Conocimientos:

- La Legislación Universitaria y en particular el Estatuto del Personal Académico.

- Marco jurídico nacional en materia de salud.

- El plan de estudios por competencias y sus programas académicos.

- Competencias del perfil del licenciado y el perfil profesional.

- Estructura orgánica y funcional de la Facultad de Medicina y su vinculación con otras instituciones de educación superior.

- El contexto institucional universitario

- Organización del Sistema Nacional de Salud y su vinculación con la Facultad de Medicina.

- Fortalezas, debilidades, áreas de oportunidad del ejercicio médico en los sectores público y privado.

- Procedimientos de la administración escolar de la Facultad de Medicina.

b) Habilidades:

- Orientar al estudiante en la solución de problemas académico-administrativos.

\section{Humanística}

a) Conocimientos:

- Dimensión humanística de la medicina.

- Generalidades sobre humanidades médicas.

- Dimensión ética del acto médico.

- Papel de la reflexión bioética en el ejercicio de la profesión médica.

- Problemática socioeconómica, política y cultural a la que se enfrenta el médico en el mundo actual.

b) Habilidades:

Crear condiciones óptimas para:

- La reflexión filosófica en medicina.

- La formulación de juicios de valor.

- La valoración de los logros de la medicina en tiempo y espacio.

- La toma de conciencia sobre los problemas éticos de la medicina contemporánea.

- La comprensión de la naturaleza plural de la sociedad. 


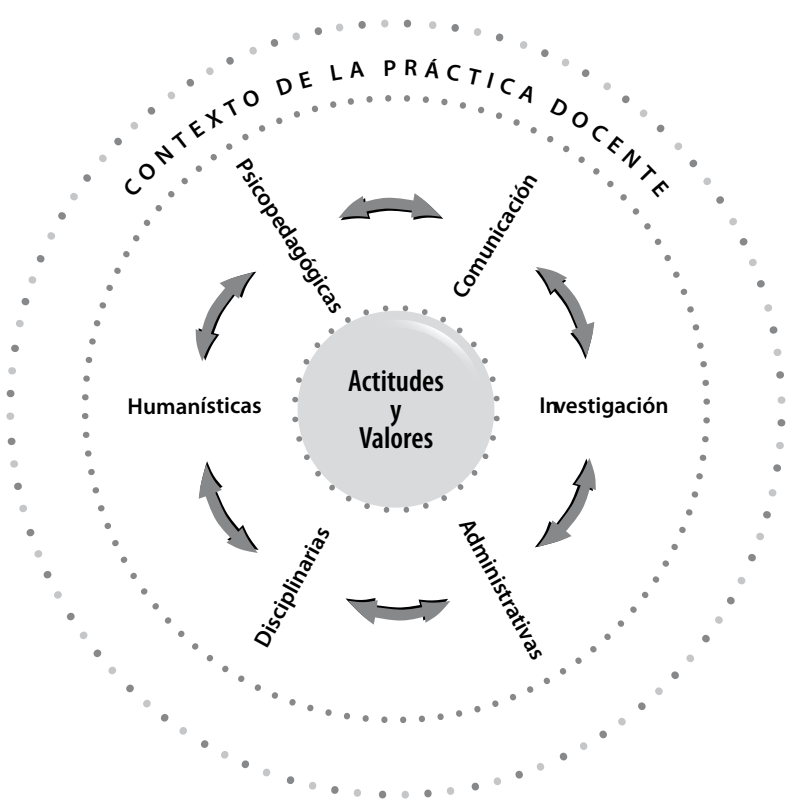

Figura. Modelo de competencias de un profesor de medicina.

\section{Tabla III. Perfil de competencias del profesor de medicina (actitudes y valores).}

- Compromiso con los fines de la institución

- Sentido de pertenencia institucional

- Compromiso con los estudiantes

- Autocrítica

- Heterocrítica

- Perseverancia

- Asertividad

- Ecuanimidad

- Flexibilidad

- Respeto

- Tolerancia

- Entusiasmo

- Calidez

- Empatía

- Conciliación

- Apertura

- Receptividad

- Prudencia

- Discreción

- Reflexión

- Previsión

- Responsabilidad

- Organización tran aquellos que demandan una visión del desempeño docente más coherente con una visión integral, como es el caso del modelo por competencias aquí presentado. La concepción rutinaria $y$ tradicional del profesor como un 'expositor de clases', donde éste es el principal actor del proceso de enseñanza-aprendizaje, constituye para las necesidades actuales de formación de médicos y de los profesores correspondientes, un modelo atípico e insuficiente, que reclama una participación central, comprometida y dinámica del estudiante en su proceso de formación profesional.

Diversos autores [8-11] reconocen la importancia de que el profesor cambie su metodología de enseñanza, al aprendizaje centrado en el alumno. Los cursos que describen para la formación de profesores incluyen conocimientos, habilidades, actitudes y valores similares a los que se señalan en el modelo aquí planteado. La diferencia estriba en que estos autores no contemplan la formación integral basada en competencias ni una metodología como la que se utilizó, en este trabajo, para identificarlas [21].

Otros autores $[6,8]$ señalan tópicos que se deben tratar, que coinciden con los resultados aquí obtenidos con relación a métodos de enseñanza, evaluación del aprendizaje e investigación educativa. También muestran semejanza en cuanto a 
que el maestro es un modelo que hay que seguir, proveedor de información y administrador, es decir, las funciones y actividades que debe realizar un profesor de medicina. Sin embargo, no describen las competencias necesarias para llevar a cabo estas funciones.

Nogueira et al [5] proponen diez competencias que corresponden, desde el punto de vista de los autores de este artículo, a funciones y actividades que incluyen áreas como las disciplinarias, pedagógicas, humanísticas, administrativas y de comunicación. Es de hacer notar que éstos no incluyen las áreas psicológica y de investigación.

En este trabajo se identificaron y organizaron las funciones, actividades, el perfil y el modelo de competencias de los profesores de medicina, derivados del análisis de la bibliografía, del propósito del plan de estudio y con apoyo del método DACUM. Las ocho funciones descritas están estrechamente vinculadas y pretenden en su conjunto una educación integral, con mayor énfasis en los aspectos formativos. Las 49 actividades básicas requeridas para que los profesores cubran satisfactoriamente las funciones establecidas, puntualizan la conveniencia de una interacción cotidiana entre el profesor y el alumno.

El perfil de competencias establecido en este estudio (Tablas II y III) revela un alto grado de exigencia para los profesores de medicina. Si la función primaria de todos ellos es guiar al estudiante en el desarrollo de su formación como médico y sugerir mejoras oportunas para garantizar la calidad, es evidente que debe ser un profesional altamente calificado y con convicción que permita juzgar el nivel de su preparación, creatividad, interés en la docencia, habilidad para conducir a los estudiantes y conocimiento acerca de los aspectos administrativos de la institución asociados a la docencia. Este perfil es un requisito fundamental y uno de los factores determinantes en el camino a la excelencia académica, ya que la formación al lado de profesores con esas características propicia que los titulados tengan éxito en el ejercicio profesional.

Tradicionalmente se selecciona y contrata a los profesores en base a su competencia disciplinaria, sin dar la debida importancia a las cinco competencias restantes aquí señaladas $y$, como otros autores han sugerido, esto no es suficiente para garantizar un profesional apto para la docencia $[6,16]$, por lo que es necesario profesionalizarla.

El modelo generado (Figura) representa una estructura abierta, dinámica y flexible. Abierta, porque permite el movimiento de entrada y salida de múltiples influencias dentro del contexto de la práctica docente. Dinámica, en función de que cada una de estas competencias está estrechamente articulada a las demás y esta vinculación hace posible su interacción. Flexible, debido a que cada una de las competencias tiene diferente relevancia según el contexto de la práctica docente, sustentada en actitudes y valores epistémicos y éticos.

Si se tiene en cuenta el papel clarificador y facilitador que esta estructura desempeña en el proceso de resolución de problemas en un ámbito del saber, saber hacer y ser, como es la educación médica, la intención del modelo es:

- Proporcionar elementos para seleccionar a los profesores que evidencien poseer las competencias académicas.

- Guiar las acciones de los profesores que actualmente participan en el programa y de aquellos que aspiran a formar parte del proceso educativo.

- Elaborar programas para la formación de profesores que favorezcan el desarrollo de competencias académicas.

- Identificar elementos que se deben considerar para la evaluación del desempeño docente.

- Orientar el diseño de indicadores útiles para otorgar reconocimientos y estímulos a los profesores cuyo desempeño académico sea excepcional.

- Orientar al estudiante, en la relación profesoralumno, sobre las funciones y actividades que se puede esperar del profesor.

En conclusión, el modelo generado define las competencias básicas que debieran tener quienes se dedican a la docencia en medicina. Sin embargo, muchas de las competencias son comunes a otras disciplinas y a otros niveles de estudio [24,25]. Este modelo puede ser aplicado a otros programas de ciencias de la salud, y de aquí se puede desprender una línea de investigación para establecer un modelo de competencias genéricas, aplicables a todos los profesores universitarios. 


\section{Bibliografía}

1. Franks NR, Richardson T. Teaching in tandem-running ants. Nature 2006; 439: 153-8.

2. Biggs J. What the student does: teaching for enhanced learning. Higher Educ Res Dev 1999; 18: 57-75.

3. Modell HI. Evolution of an educator: lessons learned and challenges ahead. Adv Physiol Educ 2004; 28: 88-94.

4. Department of education of Australia. Principles of effective learning and teaching. Brisbane: Queensland Dept. of Education; 1994.

5. Nogueira SM, Rivera MC, Blanco HF. Competencias docentes del médico de familia en el desempeño de la tutoría en la carrera de medicina de la Facultad de Ciencias médicas Julio Trigo López, Ciudad de la Habana, Cuba. Educ Med Sup 2005; 19: 11-21.

6. Harden RM, Crosby J. AMEE guide No. 20: the good teacher is more than a lecturer -the twelve roles of the teacher. Med Teach 2000; 22: 334-7.

7. Zabar S, Hanley K, Stevens DL. Measuring the competence of residents as teachers. J Gen Intern Med 2004; 19: 530-3.

8. Gruppen LS, Simpson D, Searle N. Educational fellowship programs: common themes and overarching issues. Acad Med 2006; 11: 990-4.

9. Hattem CJ, Lown BA, Newman LR. The academia health center coming of age: helping faculty become better teachers and agents of educational change. Acad Med 2006; 11: 941-4.

10. Muller JH, Irby DM. Developing educational leaders: the teaching scholars program at the University of California, San Francisco, School of Medicine. Acad Med 2006; 11: 936-40.

11. Searle NS, Hetem CH, Perkowsky L, Wilferson L. Why invest in an educational fellowship program. Acad Med 2006; 81:936-40.

12. Ramani $\mathrm{S}$. Twelve tips to promote excellence in medical teaching. Med Teach 2006; 28: 19-23.
13. General Medical Council. The doctor as teacher. London; 1999.

14. Amin Z, Eng KH, Gwee M, Hoon TC, Rhoon KD. Addressing the needs and priorities of medical teachers through a collaborative intensive faculty development programme. Med Teach 2006; 28: 85-8.

15. Comisión revisora del plan de estudios. Nueva orientación del plan de estudios de la carrera de médico cirujano de la Facultad de Medicina de la UNAM. I Congreso Académico Dr. Roberto Kretschmer. Facultad de Medicina, UNAM. México, D.F., 22 de abril de 2005.

16. Pérez-Rodríguez BA, Viniegra-Velázquez L. La formación de profesores de medicina. Comparación de dos estrategias educativas en el aprendizaje de la crítica de la información. Rev Invest Clin 2003; 55: 281-8.

17. Hesse M. Models and analogies in science. Notre Dame: University of Notre Dame Press; 1966.

18. Black M. Models and archetypes. In Black M, ed. Models and metaphor. Ithaca: Cornell University Press; 1962.

19. Norton RE. DACUM handbook. Columbus: National Centre for Vocational Education; 1985.

20. Steiner GA. Planeación estratégica: lo que todo directivo debe saber. México: CECSA; 1994.

21. Coffin L. Manual del facilitador DACUM. México: Canadian Vocational Association; 1995.

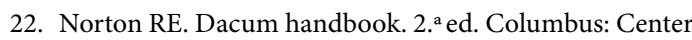
on education and training for employment; 1997.

23. Van der Klink M, Boon J. The investigation of competencies within professional domains. HRDI 2002; 5: 411-24.

24. García MC, Laguna J, Campos JO, Ruiz R, Martínez A. Competencias académicas de los tutores del posgrado en ciencias de la tierra de la UNAM. GEOS 2001; 21: 47-54.

25. Martínez A, Laguna J, Romero R, Ruiz R, García MC. Competencias académicas de los tutores de maestría y doctorado en historia de la UNAM. Históricas 2001; 62: 29-39. 
